\title{
Hölder Continuity of the Green Function and Markov Brothers' Inequality
}

\author{
Mirosław Baran • Leokadia Bialas-Ciez
}

Received: 8 March 2013 / Revised: 1 July 2013 / Accepted: 5 September 2013 /

Published online: 13 December 2013

(C) The Author(s) 2013. This article is published with open access at Springerlink.com

\begin{abstract}
Let $V_{E}$ be the pluricomplex Green function associated with a compact subset $E$ of $\mathbb{C}^{N}$. The well-known Hölder continuity property of $E$ means that there exist constants $B>0, \gamma \in(0,1]$ such that $V_{E}(z) \leq B \operatorname{dist}(z, E)^{\gamma}$. The main result of this paper says that this condition is equivalent to a Vladimir Markov-type inequality; i.e., $\left\|D^{\alpha} P\right\|_{E} \leq M^{|\alpha|}(\operatorname{deg} P)^{m|\alpha|}(|\alpha| !)^{1-m}\|P\|_{E}$, where $m, M>0$ are independent of the polynomial $P$ of $N$ variables. We give some applications of this equivalence, e.g., for convex bodies in $\mathbb{R}^{N}$, for uniformly polynomially cuspidal sets and for some disconnect compact sets.
\end{abstract}

Keywords Pluricomplex Green's function · Hölder continuity property ·

Markov inequality

Mathematics Subject Classification (2010) 41A17 · 32U35

\section{Introduction}

Let $E$ be a compact set in $\mathbb{C}^{N}$. The pluricomplex Green's function (with pole at infinity) of $E$ can be defined by

$$
V_{E}(z):=\sup \left\{u(z): u \in \mathcal{L}_{N} \text { and } u \leq 0 \text { on } E\right\}, \quad z \in \mathbb{C}^{N},
$$

Communicated by Vilmos Totik.

M. Baran · L. Bialas-Ciez ( $\varangle)$

Faculty of Mathematics and Computer Science, Institute of Mathematics, Jagiellonian University, Łojasiewicza 6, 30-348 Kraków, Poland

e-mail: Leokadia.Bialas-Ciez@im.uj.edu.pl

M. Baran

e-mail: Miroslaw.Baran@im.uj.edu.pl 
where $\mathcal{L}_{N}$ is the Lelong class of all plurisubharmonic functions in $\mathbb{C}^{N}$ of logarithmic growth at the infinity; i.e.,

$$
\mathcal{L}_{N}:=\left\{u \in \operatorname{PSH}\left(\mathbb{C}^{N}\right): u(z)-\log \|z\|_{2} \leq \mathcal{O}(1) \text { as }\|z\|_{2} \rightarrow \infty\right\}
$$

(for background information, see [15]). Here $\|z\|_{2}$ stands for the Euclidean norm in $\mathbb{K}^{N}, \mathbb{K}=\mathbb{C}$ or $\mathbb{K}=\mathbb{R}$. In the univariate case, $V_{E}$ coincides with Green's function $g_{E}$ of the unbounded component of $\hat{\mathbb{C}} \backslash E$ with logarithmic pole at infinity (as usual, $\hat{\mathbb{C}}=\mathbb{C} \cup\{\infty\})$.

If $V_{E}^{*}(z)$ is the standard upper regularization of $V_{E}$, then it is well known (Siciak's theorem), that either $V_{E}^{*} \in \mathcal{L}_{N}$ or $V_{E}^{*} \equiv+\infty$. It is also equivalent to the fact that $E$ is a nonpluripolar or pluripolar set, respectively. If we define the $L$-capacity of $E$ as $C(E)=\liminf _{\|z\|_{2} \longrightarrow \infty} \frac{\|z\|_{2}}{\exp V_{E}^{*}(z)}$, then $E$ is a pluripolar set if and only if $C(E)=0$.

A set $E$ is L-regular if $\lim _{w \rightarrow z} V_{E}^{*}(w)=0$ for every $z \in E$. Siciak has proved that this is equivalent to the continuity of $V_{E}$ in the whole space $\mathbb{C}^{N}$. Therefore, L-regularity is one of the global properties of $E$, and a crucial role is played here by the continuity of $V_{E}$ near $E$.

Another global property of the set $E$ that depends only on the behavior of $V_{E}$ near $E$ is the Hölder continuity property (HCP for short) of the pluricomplex Green's function $V_{E}$ (see the result due to Błocki in [29, Proposition 3.5]). By Cauchy's inequality, one can prove that HCP implies the A. Markov inequality; i.e., there exist constants $m \geq 1, M>0$ such that for every polynomial $P$ of $N$ variables,

$$
\|\operatorname{grad} P\|_{E} \leq M(\operatorname{deg} P)^{m}\|P\|_{E},
$$

where $\operatorname{deg} P$ is the total degree of the polynomial $P$, i.e., the highest degree of its monomials. If $E$ admits inequality (1), then it is said to be a Markov set, and we write $E \in \operatorname{AMI}(m, M)$. To reveal the importance of this property, we refer to a theorem due to Pleśniak (see [25, Theorem 3.3]).

An exciting question is whether there exists a relationship between the A. Markov inequality and the behavior of Green's function near the considered set. It is known (see [7]) that every Markov set $E \subset \mathbb{C}$ is not polar and $E$ is L-regular if $E \subset \mathbb{R}$ ([9]). It seems that the A. Markov inequality (1) implies the Hölder continuity property, but a proof is an open problem mentioned, e.g., in [25]. Actually, even the question about L-regularity of Markov sets in the general case remains open.

We shall make an attempt in the direction of solving this problem by concentrating on a generalization of an inequality proved by A. Markov's younger brother, V. Markov. He discovered in 1892, after a very detailed investigation, a precise but intricate estimate for the $k$-th derivative of polynomials (see, e.g., [27]): for any polynomial $P$ of degree not greater than $n$,

$$
\left\|P^{(k)}\right\|_{[-1,1]} \leq T_{n}^{(k)}(1)\|P\|_{[-1,1]}=\frac{n^{2}\left[n^{2}-1\right] \cdots\left[n^{2}-(k-1)^{2}\right]}{1 \cdot 3 \cdots(2 k-1)}\|P\|_{[-1,1]},
$$

where $T_{n}(x)=\cos (n \arccos x)$ is the $n$-th Chebyshev polynomial (for $k=1$, it was proved by A. Markov in 1889).

Inequality (2) inspired us to consider a new type of Markov inequality (see Definition 2.5 below). It turns out that this inequality is equivalent to the Hölder continuity 
property of the pluricomplex Green function. This is the main result of the paper (Theorem 2.9).

Although the definition of HCP is simple, its verification for particular sets can be very complicated (see, e.g., [1, 16-19, 26]). The Carleson-Totik criterion (see [13, Theorems 1.2 and 1.7]) merits mentioning here. It gives an equivalent condition for HCP expressed in terms of capacities in a similar way to Wiener's criterion for L-regularity. This criterion can be used for proving HCP for a large family of sets. However, the Carleson-Totik criterion holds only in the univariate complex case (or in $\mathbb{R}^{N}$ ), and the equivalence is valid under certain additional assumptions on sets, e.g., for sets satisfying an exterior cone condition. In this context, Theorem 2.9 of this paper provides a useful tool for showing HCP, especially when sets do not satisfy the assumptions of the criterion mentioned above. We give some examples of such an application of Theorem 2.9. Moreover, we prove a rather surprising fact that it is sufficient to verify the Hölder continuity property of $V_{E}$ only in $N$ canonical directions (Corollary 2.10). This allows us to show HCP for a large class of sets.

The paper is organized as follows. A statement of the main results is presented in Sect. 2. The next section contains proofs of these results. In Sect. 4, we give some applications of Theorem 2.9 for compact subsets of $\mathbb{R}^{N} \subset \mathbb{R}^{N}+i \mathbb{R}^{N}=\mathbb{C}^{N}$, especially with convex bodies and also with UPC sets, i.e., uniformly polynomially cuspidal sets. In the last section, we show some applications of Theorem 2.9 for disconnected compact sets.

\section{Notation and Statement of the Main Results}

The pluricomplex Green's function is closely related to polynomials (see [28] or [15, Theorem 5.1.7]) in view of the formulas

$$
V_{E}(z)=\log \Phi_{E}(z), \quad z \in \mathbb{C}^{N},
$$

where $\Phi_{E}$ is the Siciak extremal function; i.e.,

$$
\Phi_{E}(z)=\sup \left\{|P(z)|^{1 / n} /\|P\|_{E}^{1 / n}: P \in \mathcal{P}\left(\mathbb{C}^{N}\right), \operatorname{deg} P=n \geq 1,\left.P\right|_{E} \not \equiv 0\right\},
$$

$\mathcal{P}\left(\mathbb{K}^{N}\right)$ denotes the vector space of polynomials of $N$ variables with coefficients in $\mathbb{K} \in\{\mathbb{C}, \mathbb{R}\}$, and $\|\cdot\|_{E}$ is the maximum norm on $E$.

In order to investigate the behavior of $V_{E}$ near $E$, we define

$$
V_{E}^{\bullet}(z):=\sup \left\{V_{E}(x-w): x \in E,\|w\|_{2} \leq\|z\|_{2}\right\}, \quad z \in \mathbb{C}^{N},
$$

that is, a radial modification of $V_{E}$. The definition and main properties of $V_{E}^{\bullet}$ were presented by M. Baran, L. Bialas-Ciez, Comparison principles for compact sets in $\mathbb{C}^{N}$ with HCP and Markov properties during the Conference on Several Complex Variables on the occasion of Professor's Józef Siciak's 80th birthday, Kraków, 4-8 July 2011. We set out (without proofs) the following examples:

- if $E$ is a unit ball in $\mathbb{C}^{N}$ (with respect to a fixed complex norm), then $V_{E}(z)=$ $\max \{0, \log \|z\|\}$ and $V_{E}^{\bullet}(z)=\log \left(1+\|z\|_{2} / C(E)\right)$, 
- if $E$ is the real unit Euclidean ball in $\mathbb{R}^{N}$, then $V_{E}(z)=\frac{1}{2} \log h\left(\|z\|^{2}+\left|z^{2}-1\right|\right)$, where $z^{2}=z_{1}^{2}+\cdots+z_{N}^{2}$ for $z=\left(z_{1}, \ldots, z_{N}\right)$ and $h(t)=t+\sqrt{t^{2}-1}$ for $t \geq 1$,

- if $E$ is a convex symmetric body in $\mathbb{R}^{N}$, then $V_{E}^{\bullet}(z)=\log h\left(1+\|z\|_{2} /(2 C(E))\right)$,

- if $E$ is a pluripolar set, then $V_{E}^{\bullet}(0)=0,\left.V_{E}^{\bullet}\right|_{\mathbb{C}^{N} \backslash\{0\}} \equiv+\infty$.

For the nonpluripolar sets we can obtain a very important fact which is derived from Proposition 1.4 in [20] (cf. [5, Theorem 2.1c)]):

Theorem 2.1 If E is a nonpluripolar compact subset of $\mathbb{C}^{N}$ and

$$
\rho_{E}(r):=V_{E}^{\bullet}(z) \quad \text { for }\|z\|_{2}=r,
$$

then $t \longmapsto \rho_{E}\left(e^{t}\right)$ is an increasing convex function.

Remark 2.2 The function $\rho_{E}$ has the following basic properties:

(a) $\rho_{\lambda E}(r)=\rho_{E}\left(\lambda^{-1} r\right), \lambda>0$,

(b) $\rho_{E \times F}(r)=\max \left(\rho_{E}(r), \rho_{F}(r)\right)$,

(c) $\lim _{r \rightarrow \infty}\left(\rho_{E}(r)-\log r\right)=-\log C(E)$,

(d) if $E$ is L-regular, then $\left\|V_{E}\right\|_{E_{r}}=\rho_{E}(r)$, where $E_{r}=\left\{z \in \mathbb{C}^{N}: \operatorname{dist}(z, E) \leq r\right\}$,

(e) $\rho_{E}$ is increasing, continuous on $(0,+\infty)$, and consequently, $0=$ $\rho_{E}(0) \leq \lim _{r \rightarrow 0+} \rho_{E}(r)$. Therefore, L-regularity is equivalent to the equality $\lim _{r \rightarrow 0+} \rho_{E}(r)=0$.

Indeed, equality (a) can be checked by a standard verification. Formula (b) is a consequence of the well-known product property of the pluripotential Green function. A behavior of $\rho_{E}$ for $r$ near infinity is related to the L-capacity of $E$ : as in the proof of Theorem 2.3 in [5], we can show equality (c). Statements (d) and (e) are deduced directly from Theorem 2.1.

We are interested in seeing how the Hölder continuity of the pluricomplex Green's function $V_{E}$ is connected with Markov-type inequalities for polynomials on $E$. The question will be made more precise by the next definition:

Definition 2.3 Let $\gamma \in(0,1], B>0$. A compact set $E \subset \mathbb{C}^{N}$ admits the Hölder continuity property of the pluricomplex Green's function $V_{E}(E \in H C P(\gamma, B)$ for short) if for every $z \in \mathbb{C}^{N}$,

$$
V_{E}(z) \leq B \operatorname{dist}(z, E)^{\gamma}
$$

By Błocki’s argument (see [29, Proposition 3.5]), property (4) implies the Hölder continuity with the same exponent $\gamma$ of the pluricomplex Green's function $V_{E}$ in the whole space.

If $E \in H C P(\gamma, B)$, then $E$ is $L$-regular, and therefore $C(E)>0$. However, a lower bound for $C(E)$ in terms of the constants $\gamma, B$ was not known. In this paper, we simply solve this problem (see Theorem 2.9).

Stirling's formula and Theorem 3.5 in [21] applied to the polynomial $P(x)=x$ lead us to: 
Proposition 2.4 If $E \subset \mathbb{C}, n$ is an integer positive number, $k \in\{1, \ldots, n\}$, and there exists $M_{k}=M_{k}(E), m>0$ such that for every polynomial $P$ of degree at most $n$,

$$
\left\|P^{(k)}\right\|_{E} \leq M_{k} n^{m k}\|P\|_{E},
$$

then $M_{k} \geq B^{k} /\left[(k !)^{m-1}\right]$ for a certain constant $B>0$ depending only on the set $E$.

This fact was the motivation for concentrating on the following generalization of the V. Markov inequality.

Let $\mathbb{N}=\{1,2, \ldots\}$ and $\mathbb{N}_{0}=\mathbb{N} \cup\{0\}$.

Definition 2.5 Fix $m \geq 1, M>0$. A compact set $E \subset \mathbb{C}^{N}$ admits the $V$. Markov inequality $\left(E \in \operatorname{VMI}(m, M)\right.$ for short) if for every $\alpha \in \mathbb{N}_{0}^{N}, P \in \mathcal{P}\left(\mathbb{C}^{N}\right)$,

$$
\left\|D^{\alpha} P\right\|_{E} \leq M^{|\alpha|} \frac{(\operatorname{deg} P)^{m|\alpha|}}{(|\alpha| !)^{m-1}}\|P\|_{E},
$$

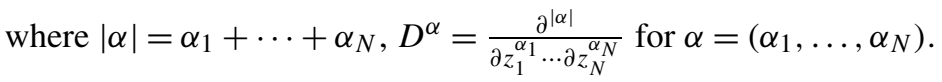

In other words, (6) is a version of inequality (5) (and also its analog in higher dimensional space) with the strongest possible constants $M_{k}$ (compare with [6], where best Markov exponents were studied).

Example 2.6 The simplest example of a set admitting V. Markov inequality is the unit disk $\overline{\mathbb{D}}$ in the complex plane. By the Bernstein inequality, for every polynomial $P$ of degree at most $n$, we have $\left\|P^{(k)}\right\|_{\overline{\mathbb{D}}} \leq \frac{n !}{(n-k) !}\|P\|_{\overline{\mathbb{D}}}$, and thus $\overline{\mathbb{D}} \in \operatorname{VMI}(1,1)$. In the multidimensional space for a polydisk $D(a, r)=\left\{z \in \mathbb{C}^{N}:\left|z_{1}-a_{1}\right| \leq r_{1}, \ldots, \mid z_{N}-\right.$ $\left.a_{N} \mid \leq r_{N}\right\}$ of polyradius $r=\left(r_{1}, \ldots, r_{N}\right) \in(0,+\infty)^{N}$, we get (see [8, Example 2.2])

$$
\left\|D^{\alpha} P\right\|_{D(a, r)} \leq \frac{v !}{(v-\alpha) ! r^{\alpha}}\|P\|_{D(a, r)}
$$

whenever $P$ is a polynomial of $N$ variables $z_{1}, \ldots, z_{N}$ of degree at most $v_{1}$ in $z_{1}, \ldots, v_{N}$ in $z_{N}$. As usual, $v !=v_{1} ! \cdots v_{N} !$ and $r^{\alpha}=r_{1}^{\alpha_{1}} \cdots r_{N}^{\alpha_{N}}$. Hence,

$$
\left\|D^{\alpha} P\right\|_{D(a, r)} \leq r^{-\alpha} v_{1}^{\alpha_{1}} \cdots v_{N}^{\alpha_{N}}\|P\|_{D(a, r)} \leq r^{-\alpha}(\operatorname{deg} P)^{|\alpha|}\|P\|_{D(a, r)}
$$

for any polynomial $P \in \mathcal{P}\left(\mathbb{C}^{N}\right)$. Therefore, $D(a, r) \in \operatorname{VMI}\left(1, \max _{j} 1 / r_{j}\right)$.

Example 2.7 Due to the classical inequality proved by V. Markov (see (2)), we have $[-1,1] \in \operatorname{VMI}(2,1)$. If $E=\left[a_{1}, b_{1}\right] \times \cdots \times\left[a_{N}, b_{N}\right] \subset \mathbb{R}^{N} \subset \mathbb{R}^{N}+i \mathbb{R}^{N}=\mathbb{C}^{N}$, then for every polynomial $P$ of degree at most $v_{1}$ in $z_{1}, \ldots, v_{N}$ in $z_{N}$, we have (see [8, Example 2.2])

$$
\left\|D^{\alpha} P\right\|_{E} \leq \frac{2^{|\alpha|}}{(b-a)^{\alpha}} T_{v_{1}}^{\left(\alpha_{1}\right)}(1) \cdots T_{v_{N}}^{\left(\alpha_{N}\right)}(1)\|P\|_{E} \leq \frac{2^{|\alpha|}}{(b-a)^{\alpha}} \cdot \frac{v^{2 \alpha}}{\alpha !}\|P\|_{E},
$$


with $a=\left(a_{1}, \ldots, a_{N}\right), b=\left(b_{1}, \ldots, b_{N}\right), v=\left(v_{1}, \ldots, v_{N}\right)$. Since $N^{|\alpha|} \alpha ! \geq|\alpha|$ !, we obtain $E \in \operatorname{VMI}\left(2,2 N \max _{j} 1 /\left(b_{j}-a_{j}\right)\right)$.

It is evident that $\operatorname{VMI}(m, M) \Rightarrow \operatorname{AMI}(m, M \sqrt{N})$. On the other hand, property (1) easily implies that

$$
\left\|D^{\alpha} P\right\|_{E} \leq M^{|\alpha|}\left(\frac{n !}{(n-|\alpha|) !}\right)^{m}\|P\|_{E} \leq M^{|\alpha|} n^{m|\alpha|}\|P\|_{E}
$$

for any $\alpha \in \mathbb{N}_{0}^{N}, P \in \mathcal{P}\left(\mathbb{C}^{N}\right)$ of degree at most $n$.

Remark 2.8 If $E \in \operatorname{AMI}\left(m_{1}, M_{1}\right)$, and if we fix an arbitrary $\delta \in(0,1)$, then for every polynomial $P$ of degree at most $n$ and for all $|\alpha| \leq n^{\delta}$, inequality (6) holds with $m=\frac{m_{1}-\delta}{1-\delta}$ and $M=M_{1}$. Indeed,

$$
\left\|D^{\alpha} P\right\|_{E} \leq\left(M_{1} n^{m_{1}}\right)^{|\alpha|}\|P\|_{E},
$$

and

$$
n^{m_{1}|\alpha|}=\left(\frac{n^{m_{1}}|\alpha|^{m-1}}{|\alpha|^{m-1}}\right)^{|\alpha|} \leq \frac{\left(n^{m_{1}+(m-1) \delta}\right)^{|\alpha|}}{|\alpha|^{(m-1)|\alpha|}} \leq \frac{n^{m|\alpha|}}{|\alpha|^{m-1}} .
$$

By the above, in the particular case of $m_{1}=1$, we get $\operatorname{AMI}\left(1, M_{1}\right) \Leftrightarrow \operatorname{VMI}(1, M)$.

In the general case, we do not know whether or not the V. Markov inequality is equivalent to that of A. Markov. However, we can show that the Hölder continuity property is equivalent to (6).

Theorem 2.9 (Main theorem) If $E$ is a compact subset of $\mathbb{C}^{N}, 0<\gamma \leq 1 \leq m$, $B, M>0$, then

$$
\begin{aligned}
& E \in \operatorname{HCP}(\gamma, B) \Longrightarrow E \in \operatorname{VMI}(m, M), \quad \text { with } m=1 / \gamma, M=\sqrt{N}(B \gamma e)^{1 / \gamma}, \\
& E \in \operatorname{VMI}(m, M) \Longrightarrow E \in \operatorname{HCP}(\gamma, B), \quad \text { with } \gamma=1 / m, B=M^{\gamma} N^{\gamma} m .
\end{aligned}
$$

Moreover, if $E \in \operatorname{VMI}(m, M)$, then $C(E) \geq e^{-m} \frac{1}{N M}$. Hence, if $E \in \operatorname{HCP}(\gamma, B)$, then $C(E) \geq\left(N^{3 / 2}\left(B \gamma e^{2}\right)^{1 / \gamma}\right)^{-1}$.

As a consequence of the above theorem, the well-known open problem concerning the conjectured implication $\mathrm{AMI} \Rightarrow \mathrm{HCP}$ is equivalent to a new question of whether AMI implies VMI. The first problem concerns the properties related to the notions in two different fields, the pluricomplex Green's function and polynomials, whereas the new question is formulated only in terms of derivatives of polynomials.

Due to the above theorem, we can give new, somewhat unexpected equivalents to the Hölder continuity property of the pluricomplex Green's function:

Corollary 2.10 If $E$ is a compact subset of $\mathbb{C}^{N}$ and $\gamma \in(0,1]$, then the following conditions are equivalent: 
(i) $E \in H C P\left(\gamma, B_{1}\right)$, with some $B_{1} \geq 1$,

(ii) $\exists B_{2}>0 \forall z_{0} \in E \forall j \in\{1, \ldots, N\} \forall \zeta \in \mathbb{C}$ such that $|\zeta| \leq 1$ we have

$$
V_{E}\left(z_{0}+\zeta e_{j}\right) \leq B_{2}|\zeta|^{\gamma}
$$

(iii) $\exists M_{3}>0 \forall j \in\{1, \ldots, N\} \forall P \in \mathcal{P}\left(\mathbb{C}^{N}\right) \forall k \in \mathbb{N}$ we have

$$
\left\|D^{k e_{j}} P\right\|_{E} \leq M_{3}^{k} \frac{(\operatorname{deg} P)^{k / \gamma}}{k !^{\frac{1}{\gamma}}-1}\|P\|_{E},
$$

where $e_{1}, \ldots, e_{N}$ are the canonical vectors in $\mathbb{C}^{N}: e_{j}=(0, \ldots, 0,1,0, \ldots, 0)$ with the value 1 in the $j$-th entry.

It seems to be rather surprising that condition (ii) in Corollary 2.10, which holds only in $N$ canonical directions, is sufficient to guarantee the Hölder continuity property of $V_{E}$ in all directions.

For the compact subsets of $\mathbb{R}^{N} \subset \mathbb{R}^{N}+i \mathbb{R}^{N}=\mathbb{C}^{N}$, we prove that if inequality (4) holds for $x \in \mathbb{R}^{N}$, then it also holds for all $z \in \mathbb{C}^{N}$ (Corollary 4.4). As a consequence, we obtain that $E \in \operatorname{HCP}(1 / 2, \mathcal{B} / \sqrt{C(E)})$ for any convex body in $\mathbb{R}^{N}$, where $C(E)$ is the L-capacity of $E$ and $\mathcal{B}$ is an absolute constant independent of $E$ and even of $N$ (Example 4.7). Moreover, we prove that every set $E \subset \mathbb{R}^{N}$ uniformly polynomially cuspidal in direction $v$ with exponent $s$ has the following property: $V_{E}(x+\zeta v) \leq$ $B|\zeta|^{1 /(2 s)}$ for $x \in E,|\zeta| \leq r_{0}$ (Theorem 4.10). Hence we deduce from Corollary 2.10 that every UPC compact subset of $\mathbb{R}^{N}$ admits HCP, and thus V. Markov inequality (Corollary 4.11). In this way, we obtain a wide class of sets that have such a property. This is the first essential generalization of V. Markov's result from the end of the 19th century.

As another application of the main theorem, we can prove HCP for disconnected sets. Proposition 5.1 regards some onion type sets in the complex plane that may not satisfy the assumptions of the Carleson-Totik criterion. These sets are particularly interesting in view of certain properties of compacts admitting so-called local Markov's inequality (see [10]). The second example of such an application of Theorem 2.9 concerns some compact sets consisting of infinitely many pairwise disjoint subsets of $\mathbb{C}^{N}$ (Proposition 5.2).

\section{Proofs of the Main Results}

Proof of Theorem 2.9. To show the first implication, consider an arbitrary polynomial $P \in \mathcal{P}\left(\mathbb{C}^{N}\right)$ of degree at most $n$ and $\alpha \in \mathbb{N}_{0}^{N}$. From (3) and the Cauchy integral formula, for fixed $z=\left(z_{1}, \ldots, z_{N}\right) \in E, r>0$, we can obtain

$$
\left|D^{\alpha} P(z)\right| \leq \frac{\alpha !}{(r / \sqrt{N})^{|\alpha|}}\|P\|_{D(z, r / \sqrt{N})} \leq \frac{\sqrt{N}^{|\alpha|} \alpha !}{r^{|\alpha|}}\|P\|_{E} \exp \left(n\left\|V_{E}\right\|_{D(z, r / \sqrt{N})}\right),
$$


where $D(z, r / \sqrt{N})=\left\{w \in \mathbb{C}^{N}:\left|w_{1}-z_{1}\right| \leq r / \sqrt{N}, \ldots,\left|w_{N}-z_{N}\right| \leq r / \sqrt{N}\right\}$. From (4) we have

$$
\left|D^{\alpha} P(z)\right| \leq \frac{\sqrt{N}^{|\alpha|} \alpha !}{r^{|\alpha|}}\|P\|_{E} \exp \left(n B r^{\gamma}\right),
$$

and for $r=(|\alpha| /(B \gamma n))^{m}, m=1 / \gamma$, we get

$$
\begin{aligned}
\left|D^{\alpha} P(z)\right| & \leq \frac{\sqrt{N}^{|\alpha|} \alpha !}{|\alpha|^{|\alpha| m}} n^{m|\alpha|}\|P\|_{E}(B \gamma e)^{|\alpha| / \gamma} \\
& \leq\left(\sqrt{N}(B \gamma e)^{1 / \gamma}\right)^{|\alpha|} \frac{|\alpha| !}{(|\alpha| !)^{m}} n^{m|\alpha|}\|P\|_{E},
\end{aligned}
$$

and (8) is proved.

We now proceed to show implication (9). For this purpose, observe that from (3), it is sufficient to prove

$$
|P(z)| \leq\|P\|_{E} \exp \left(M^{\gamma} N^{\gamma} m n r^{\gamma}\right)
$$

for any polynomial $P \in \mathcal{P}\left(\mathbb{C}^{N}\right)$ of degree at most $n$ and $z \in \mathbb{C}^{N} \backslash E$ such that $\operatorname{dist}(z, E)=r$. By Taylor's formula, we have

$$
|P(z)| \leq \sum_{|\alpha| \leq n} \frac{1}{\alpha !}\left|D^{\alpha} P(w)\right| r^{|\alpha|} \leq \sum_{k=0}^{n} \sum_{|\alpha|=k} \frac{1}{\alpha !} r^{|\alpha|}\left\|D^{\alpha} P\right\|_{E}
$$

whenever $w \in E$ and $\operatorname{dist}(z, E)=\|z-w\|_{2}$. From (6), the above inequality gives

$$
|P(z)| \leq\|P\|_{E} \sum_{k=0}^{n} M^{k} \frac{n^{m k}}{(k !)^{m-1}} r^{k} \sum_{|\alpha|=k} \frac{1}{\alpha !} .
$$

Since $\sum_{|\alpha|=k} 1 / \alpha !=N^{k} / k !$, for $\gamma=1 / m$, we have

$$
\begin{aligned}
|P(z)| & \leq\|P\|_{E} \sum_{k=0}^{n} M^{k} N^{k} \frac{n^{m k}}{(k !)^{m}} r^{k} \leq\|P\|_{E} \sum_{k=0}^{\infty}\left[\frac{\left(M^{\gamma} N^{\gamma} r^{\gamma} n\right)^{k}}{k !}\right]^{m} \\
& =\|P\|_{E} \mathcal{G}_{m}\left(M^{\gamma} N^{\gamma} r^{\gamma} n\right),
\end{aligned}
$$

where

$$
\mathcal{G}_{m}(x):=\sum_{k=0}^{\infty}\left(\frac{x^{k}}{k !}\right)^{m}=\left\|\left(\frac{x^{k}}{k !}\right)_{k \in \mathbb{N}_{0}}\right\|_{m}^{m}
$$

and $\|\cdot\|_{m}$ is the usual norm in the space $l_{m}$. As $\|\cdot\|_{m} \leq\|\cdot\|_{1}$, we have

$$
\mathcal{G}_{m}(x)=\left\|\left(\frac{x^{k}}{k !}\right)_{k \in \mathbb{N}_{0}}\right\|_{m}^{m} \leq\left\|\left(\frac{x^{k}}{k !}\right)_{k \in \mathbb{N}_{0}}\right\|_{1}^{m}=e^{x m}
$$


and

$$
|P(z)| \leq\|P\|_{E} \exp \left(M^{\gamma} N^{\gamma} r^{\gamma} m n\right)
$$

which gives (10).

If $w \in E$ and $\zeta \in \mathbb{C}^{N},\|\zeta\|_{2}=r$, then in a similar way as above, we get

$$
|P(w+\zeta)| \leq\|P\|_{E} \sum_{k=0}^{n} N^{k} M^{k} \frac{n^{m k}}{(k !)^{m}} r^{k} .
$$

In the case of $N M r \geq 1$, we obtain

$$
|P(w+\zeta)| \leq\|P\|_{E}(N M r)^{n}\left(\sum_{k=0}^{n} \frac{n^{k}}{k !}\right)^{m} \leq\|P\|_{E}(N M r)^{n} e^{n m} .
$$

Thus $\rho_{E}(r) \leq \log \left(N M e^{m}\right)+\log r$ for $r \geq e^{-m} \frac{1}{N M}$, and consequently (see Remark 2.2),

$$
-\log C(E)=\lim _{r \rightarrow \infty}\left(\rho_{E}(r)-\log r\right) \leq \log \left(N M e^{m}\right),
$$

and the proof is completed.

Proof of Corollary 2.10 First, we prove (ii) $\Rightarrow$ (iii). Set $p_{j}(\zeta)=P\left(z_{0}+\zeta e_{j}\right)$ for $\zeta \in \mathbb{C}, z_{0} \in E$ and for a fixed polynomial $P \in \mathcal{P}\left(\mathbb{C}^{N}\right)$ of degree at most $n$. Obviously, $\left|D^{k e_{j}} P\left(z_{0}\right)\right|=\left|p_{j}^{(k)}(0)\right|$, and by Cauchy's integral formula,

$$
\begin{aligned}
\mid D^{k e_{j} P\left(z_{0}\right) \mid} & \leq \frac{k !}{r^{k}} \max \left\{\left|p_{j}(\zeta)\right|:|\zeta|=r\right\} \\
& \leq \frac{k !}{r^{k}}\|P\|_{E} \max \left\{\exp \left(n V_{E}\left(z_{0}+\zeta e_{j}\right)\right):|\zeta|=r\right\}
\end{aligned}
$$

the last inequality being a consequence of (3). If $r=(k / n)^{1 / \gamma}$, then from (ii), we get

$$
\left\|D^{k e_{j}} P\right\|_{E} \leq k !\left(\frac{n}{k}\right)^{\frac{k}{\gamma}} e^{B_{2} k}\|P\|_{E} \leq M_{3}^{k} \frac{n^{k / \gamma}}{k ! \frac{1}{\gamma}-1}\|P\|_{E},
$$

with some positive constant $M_{3}$, and (iii) is proved.

In view of Theorem 2.9 , to show (iii) $\Rightarrow$ (i), it is sufficient to prove that (iii) implies inequality (6). Fix $\alpha=\alpha_{1} e_{1}+\cdots+\alpha_{N} e_{N} \in \mathbb{N}_{0}^{N}$. If $P$ is a polynomial of degree $n_{j}$ in $z_{j}$ where $z=\left(z_{1}, \ldots, z_{N}\right)$, we have

$$
\begin{aligned}
\left\|D^{\alpha} P\right\|_{E} & \leq M_{3}^{\alpha_{1}} \frac{n_{1}^{\alpha_{1} / \gamma}}{\alpha_{1} ! \frac{1}{\gamma}-1}\left\|D^{\alpha-\alpha_{1} e_{1}} P\right\|_{E} \\
& \leq M_{3}^{|\alpha|} \frac{n_{1}^{\alpha_{1} / \gamma} \cdots n_{N}^{\alpha_{N} / \gamma}}{\alpha !^{\frac{1}{\gamma}-1}}\|P\|_{E} \leq M_{3}^{|\alpha|} N^{|\alpha|\left(\frac{1}{\gamma}-1\right)} \frac{(\operatorname{deg} P)^{|\alpha| / \gamma}}{|\alpha| ! \frac{1}{\gamma}-1}\|P\|_{E},
\end{aligned}
$$


since $N^{|\alpha|} \alpha ! \geq|\alpha|$ !, and (i) follows.

The implication (i) $\Rightarrow$ (ii) is obvious, and the proof is completed.

Remark 3.1 It follows from the proof of Corollary 2.10 that we can replace condition $V_{E}\left(x+\zeta e_{j}\right) \leq C_{2}|\zeta|^{\gamma}$ for $|\zeta| \leq 1$ by the same but only for $|\zeta| \leq r_{0} \leq 1$. Indeed, for a fixed $r_{0} \in(0,1]$ in the proof of implication (ii) $\Rightarrow$ (iii), it is sufficient to write $r=r_{0} \cdot(k / n)^{1 / \gamma}$. We shall use this remark later.

\section{HCP of Compact Subsets of $\mathbb{R}^{N}$}

Remark 4.1 For any set $E \subset \mathbb{R}^{N}$, it is sufficient to consider only polynomials with real coefficients. Indeed, if $P \in \mathcal{P}\left(\mathbb{C}^{n}\right)$, then $P=Q+i R$, where $P, Q \in$ $\mathcal{P}\left(\mathbb{R}^{N}\right), \operatorname{deg} P=\max (\operatorname{deg} Q, \operatorname{deg} R)$, and

$\|P\|_{E}=\sup _{|\theta| \leq \pi}\|\cos \theta Q+\sin \theta R\|_{E}, \quad\left\|D^{\alpha} P\right\|_{E}=\sup _{|\theta| \leq \pi}\left\|D^{\alpha}(\cos \theta Q+\sin \theta R)\right\|_{E}$.

Hence, if we have the bound $\left\|D^{\alpha} P\right\|_{E} \leq C(n, k)\|P\|_{E}$ for all $P \in \mathcal{P}\left(\mathbb{R}^{N}\right), \operatorname{deg} P \leq$ $n,|\alpha| \leq n$, then the same is true for polynomials with complex coefficients.

For compact subsets of $\mathbb{R}^{N}$, we can take only real polynomials in the definition of Siciak's extremal function (cf. [2]):

$$
\Phi_{E}(z)=\sup \left\{|P(z)|^{1 / \operatorname{deg} P}: P \in \mathcal{P}\left(\mathbb{R}^{N}\right), \operatorname{deg} P \geq 1,\|P\|_{E} \leq 1\right\}, \quad z \in \mathbb{C}^{N} .
$$

The following result is a consequence of [4, Theorem 2.4].

Proposition 4.2 If $E$ is a compact subset of $\mathbb{R}^{N}$, then

$$
V_{E}(x+i y) \leq \frac{1}{\pi} \int_{-\infty}^{+\infty} V_{E}(x+t y) \frac{d t}{1+t^{2}}
$$

for every $z=x+i y \in \mathbb{C}^{N}$. Equality holds in (11) if $N=1$ (for any $z \in \mathbb{C}$ ).

As a corollary of Proposition 4.2, we obtain:

Theorem 4.3 Let $E$ be a compact set in $\mathbb{R}^{N}$. Assume that for every $x \in \mathbb{R}^{N}$, the following inequality holds:

$$
V_{E}(x) \leq B(\operatorname{dist}(x, E))^{\gamma}
$$

where $B>0, \gamma \in(0,1)$ are constants independent of $x$. Then for all $z \in \mathbb{C}^{N}$,

$V_{E}(z) \leq \widetilde{B}(\operatorname{dist}(z, E))^{\gamma}, \quad$ with $\widetilde{B}=\frac{B}{\pi} \int_{-\infty}^{+\infty}\left(1+t^{2}\right)^{\gamma / 2-1} d t=\frac{B}{\sqrt{\pi}} \frac{\Gamma(1 / 2-\gamma / 2)}{\Gamma(1-\gamma / 2)}$. 
Proof Evidently, for $z=x+i y \in \mathbb{C}^{N}$, we have

$$
\operatorname{dist}(z, E)=\left(\operatorname{dist}(x, E)^{2}+\|y\|_{2}^{2}\right)^{1 / 2} .
$$

Inequality (12) is equivalent to

$$
V_{E}(x) \leq B\left\|x-x_{0}\right\|_{2}^{\gamma} \quad \text { for all } x_{0} \in E .
$$

By Proposition 4.2, we get

$$
\begin{aligned}
V_{E}(z) & =V_{E}(x+i y) \leq \frac{1}{\pi} \int_{-\infty}^{+\infty} V_{E}(x+t y) \frac{d t}{1+t^{2}} \leq \frac{B}{\pi} \int_{-\infty}^{+\infty}\left\|x-x_{0}+t y\right\|_{2}^{\gamma} \frac{d t}{1+t^{2}} \\
& \leq \frac{B}{\pi} \int_{-\infty}^{+\infty}\left(\left\|x-x_{0}\right\|_{2}+|t|\|y\|_{2}\right)^{\gamma} \frac{d t}{1+t^{2}} \\
& \leq \frac{B}{\pi} \int_{-\infty}^{+\infty}\left(\left\|x-x_{0}\right\|_{2}^{2}+\|y\|_{2}^{2}\right)^{\gamma / 2}\left(1+t^{2}\right)^{\gamma / 2} \frac{d t}{1+t^{2}} \\
& =\widetilde{B}\left(\left\|x-x_{0}\right\|_{2}^{2}+\|y\|_{2}^{2}\right)^{\gamma / 2} .
\end{aligned}
$$

As $x_{0}$ is an arbitrary point of $E$, we obtain $V_{E}(z) \leq \widetilde{B}(\operatorname{dist}(z, E))^{\gamma}$, which completes the proof.

It may be worth reminding the reader that if a compact set $E \subset \mathbb{R}^{N}$ admits the A. Markov inequality, then the exponent $m$ in (1) is at least equal to 2 (see, e.g., [14]). Therefore, the exponent $\gamma$ in (12) may be at most equal to $\frac{1}{2}$.

Corollary 4.4 If for every $x \in \mathbb{R}^{N}$ the following inequality holds:

$$
V_{E}(x) \leq B(\operatorname{dist}(x, E))^{\gamma}
$$

with $B>0, \gamma \in\left(0, \frac{1}{2}\right]$ independent of $x$, then for all $z \in \mathbb{C}^{N}$,

$$
V_{E}(z) \leq \widetilde{B}(\operatorname{dist}(z, E))^{\gamma}, \quad \widetilde{B}=\frac{B}{\sqrt{\pi}} \frac{\Gamma(1 / 4)}{\Gamma(3 / 4)}
$$

Corollary 4.5 If $E$ is a compact subset of $\mathbb{R}^{N}$ and $\gamma \in(0,1]$, then the following conditions are equivalent:

(i) $E \in \operatorname{HCP}\left(\gamma, B_{1}\right)$, with some $B_{1} \geq 1$,

(ii) inequality (12) holds for all $x \in \mathbb{R}^{N}$, with some $B_{2} \geq 1$ independent of $x$,

(iii) $E \in \operatorname{VMI}\left(\frac{1}{\gamma}, B_{3}\right)$, with some $B_{3} \geq 1$,

(iv) inequality (6) holds, with some $B \geq 1$ for all polynomials $P$ of real coefficients. 
Example 4.6 For $E=[-1,1]$, we have $V_{E}(x)=\log h(\max (1,|x|))$, where $h(t)=$ $t+\sqrt{t^{2}-1}$ if $t \geq 1$. If $x_{0} \in E$, then

$V_{E}(x) \leq \log h\left(1+\left|x-x_{0}\right|\right)=\log \left(1+\left|x-x_{0}\right|^{1 / 2}\left(\left|x-x_{0}\right|^{1 / 2}+\left(\left|x-x_{0}\right|+2\right)^{1 / 2}\right)\right)$.

Since $\log (1+t) \leq \frac{1}{\alpha} t^{\alpha}$ for $t \geq 0,0<\alpha \leq 1$, we obtain

$$
V_{E}(x) \leq \begin{cases}(1+\sqrt{3})\left|x-x_{0}\right|^{1 / 2} ; & \left|x-x_{0}\right| \leq 1, \\ 2(1+\sqrt{3})^{1 / 2}\left|x-x_{0}\right|^{1 / 2} ; & \left|x-x_{0}\right|>1,\end{cases}
$$

hence $V_{E}(x) \leq 2(1+\sqrt{3})^{1 / 2}\left|x-x_{0}\right|^{1 / 2}$ for all $x, x_{0}$, and thus,

$$
V_{E}(x) \leq 2(1+\sqrt{3})^{1 / 2}(\operatorname{dist}(x, E))^{1 / 2} .
$$

Example 4.7 Let $E$ be a convex body in $\mathbb{R}^{N}$ that is not symmetric with respect to the origin. Fix $\xi \in S^{N-1}$, where $S^{N-1}$ is the real unit Euclidean sphere in $\mathbb{R}^{N}$. As usual, $\langle\cdot, \cdot\rangle$ is the Euclidean inner product. Set

$$
a_{\xi}(E)=\min _{x \in E}\langle x, \xi\rangle, \quad b_{\xi}(E)=\max _{x \in E}\langle x, \xi\rangle, \quad \rho_{\xi}(E)=b_{\xi}(E)-a_{\xi}(E) .
$$

The last value is called the width of $E$ in the direction $\xi$. The minimal width of $E$ is given by $\omega(E)=\inf _{\xi \in S^{N-1}} \rho_{\xi}(E)$. For $x \in \mathbb{R}^{N}$, it follows that (see [12])

$$
\begin{aligned}
V_{E}(x) & =\sup _{\xi \in S^{N-1}} V_{\left[a_{\xi}(E), b_{\xi}(E)\right]}(\langle x, \xi\rangle) \\
& =\sup _{\xi \in S^{N-1}} V_{[-1,1]}\left(2\langle x, \xi\rangle / \rho_{\xi}(E)-\left(b_{\xi}(E)+a_{\xi}(E)\right) / \rho_{\xi}(E)\right) .
\end{aligned}
$$

Therefore, in the same way as in Example 4.6, we have

$$
\begin{aligned}
V_{E}(x) & \leq \sup _{\xi \in S^{N-1}} \log h\left(1+2\left|\left\langle x-x_{0}, \xi\right\rangle\right| / \rho_{\xi}(E)\right) \leq \log h\left(1+2\left\|x-x_{0}\right\|_{2} / \omega(E)\right) \\
& \leq 2(1+\sqrt{3})^{1 / 2}\left(2\left\|x-x_{0}\right\|_{2} / \omega(E)\right)^{1 / 2}
\end{aligned}
$$

for any fixed $x_{0} \in E$, and in consequence we get

$$
V_{E}(x) \leq(2+2 \sqrt{3})^{1 / 2}(4 \operatorname{dist}(x, E) / \omega(E))^{1 / 2} \leq(2+2 \sqrt{3})^{1 / 2}(\operatorname{dist}(x, E) / C(E))^{1 / 2},
$$

where $C(E)$ is the L-capacity of $E$ and we have used the inequality $C(E) \leq \frac{1}{4} \omega(E)$ from [5, Example 3.4]. In particular, there exists an absolute constant $\mathcal{B}$ such that for all dimensions $N$ and for all convex bodies $E \subset \mathbb{R}^{N}$, the following inequality holds:

$$
V_{E}(z) \leq \mathcal{B}(\operatorname{dist}(z, E) / C(E))^{1 / 2}, \quad z \in \mathbb{C}^{N}
$$

By Theorem 2.9, we can deduce that these sets belong to $\operatorname{VMI}\left(2, \sqrt{N} \mathcal{B}^{2} e^{2} /[4 C(E)]\right)$. 
Now we recall a definition of a class of UPC sets introduced by Pawłucki and Pleśniak [22], who have shown its importance in approximation theory. In particular, they have proved a deep result (cf. [22, Corollary 6.5]) that every fat compact subanalytic subset of $\mathbb{R}^{N}$ belongs to this class (see also [23]).

Let $s \geq 1, S>0$, and $d \in\{1,2, \ldots\}$.

Definition 4.8 A compact set $E \subset \mathbb{R}^{N}$ is called uniformly polynomially cuspidal $\left(E \in \mathrm{UPC}(s, S, d)\right.$ for short) if for every $x_{0} \in E$, we can find a polynomial mapping $\varphi: \mathbb{R} \rightarrow \mathbb{R}^{N}$ of degree at most $d$ such that $\varphi(1)=x_{0}$ and

$$
\operatorname{dist}\left(\varphi(t), \mathbb{R}^{N} \backslash E\right) \geq S(1-t)^{s} \quad \text { for } t \in[0,1] .
$$

It is rather difficult to find the optimal constant $s$ in the last inequality. However, calculations are much simpler for the following modification of the above definition.

Definition 4.9 (cf. [3]) Let $v$ be a fixed unit vector in $\mathbb{R}^{N}$. A compact set $E \subset \mathbb{R}^{N}$ is called uniformly polynomially cuspidal in direction $v\left(E \in \operatorname{UPC}_{v}(s, S, d)\right.$ for short) if for every $x_{0} \in E$, we can find a polynomial mapping $\varphi: \mathbb{R} \rightarrow \mathbb{R}^{N}$ of degree at most $d$ such that $\varphi(1)=x_{0}$ and

$$
\operatorname{dist}_{v}\left(\varphi(t), \mathbb{R}^{N} \backslash E\right) \geq S(1-t)^{S} \quad \text { for } t \in[0,1] .
$$

Here $\operatorname{dist}_{v}\left(x, \mathbb{R}^{N} \backslash E\right):=\sup \{r \geq 0:[x-r v, x+r v] \subset E\}$.

If $E \in \operatorname{UPC}(s, S, d)$, then $E \in \operatorname{UPC}_{v}(s, S, d)$ for every unit vector $v$. An open problem is whether conditions $E \in \mathrm{UPC}_{v_{j}}\left(s_{j}, S_{j}, d_{j}\right), j=1, \ldots, N, v_{1}, \ldots, v_{N}$ that are linearly independent imply $E \in \operatorname{UPC}(s, S, d)$ with some $S, s, d$. It seems that this may not be true for $N \geq 3$. However, as an application of the theorem given below, we prove that if $E \in \operatorname{UPC}_{e_{j}}\left(s_{j}, S_{j}, d\right), j=1, \ldots, N$, where $\left(e_{j}\right)_{j}$ is the canonical basis, then we get $E \in \operatorname{HCP}\left(\frac{1}{2 s}\right)$, where $s=\max _{1 \leq s \leq N} s_{j}$. In particular, if $E \in \mathrm{UPC}(s, S, d)$, then $E \in \operatorname{HCP}\left(\frac{1}{2 s}\right)$, which essentially improves the earlier result by Pawłucki and Pleśniak [22, Theorem 4.1] (see also [24]). As a corollary, we get a wide class of nonconvex sets that satisfy VMI.

Theorem 4.10 If $E \in \operatorname{UPC}_{v}(s, S, d)$ and $\varepsilon_{0} \in(0,1)$, then there exists $C_{0}=C_{0}\left(\varepsilon_{0}\right)>$ 0 such that for every $|\zeta| \leq r_{0}=\frac{S}{\sqrt{2}}\left(1-\varepsilon_{0}\right)^{s}$, the following inequality holds:

$$
V_{E}\left(x_{0}+\zeta v\right) \leq C_{0}|\zeta|^{1 /(2 s)} \quad \text { for every } x_{0} \in E
$$

Proof Let $L_{0}=\sqrt{2} / S$. Set $g(\zeta)=\frac{1}{2}\left(\zeta+\zeta^{-1}\right), \widehat{g}(\zeta)=\frac{1}{2}\left(\zeta-\zeta^{-1}\right)$. For $\rho>1$, write $a=a(\rho)=((1+g(\rho)) / 2)^{-1}, b=b(\rho)=((g(\rho)-1) / 2)^{-1}, c=c(\rho)=1 / \widehat{g}(\rho)$. We have $b=a(1-a)^{-1}, c=\frac{1}{2} a(1-a)^{-1 / 2}$. Fix $\zeta_{0}=\alpha_{0}+i \beta_{0} \in \mathbb{C}^{N}$, and $x_{0}$ in $E$, and write $u_{0}=\alpha_{0} v, v_{0}=\beta_{0} v$. Define

$\psi(\zeta)=\varphi\left(a(\rho) \frac{1}{2}(g(\zeta)+1)\right)+\frac{1}{2}(g(\zeta)-1) b(\rho) u_{0}+i \widehat{g}(\zeta) c(\rho) v_{0}, \quad \zeta \in \mathbb{C},|\zeta| \geq 1$, 
where $\varphi$ is a polynomial mapping chosen to $x_{0}$ by the definition of the UPC property.

Since

$$
\begin{aligned}
\psi\left(e^{i \theta}\right) & =\varphi(a(\rho) r \tau)+(\tau-1) b(\rho) u_{0} \pm 2 \sqrt{\tau(1-\tau)} c(\rho) v_{0} \\
\text { for } \tau & =\frac{\cos \theta+1}{2}, \theta \in[0,2 \pi],
\end{aligned}
$$

we have $\psi\left(e^{i \theta}\right) \in E$ whenever

$$
(1-\tau) b(\rho)\left|\alpha_{0}\right|+2 \sqrt{\tau(1-\tau)} c(\rho)\left|\beta_{0}\right| \leq S(1-a r)^{s}, \quad \tau \in[0,1] .
$$

The last condition is satisfied if

$$
a\left(\frac{1-\tau}{1-a}\left|\alpha_{0}\right|+\sqrt{\tau \frac{1-\tau}{1-a}}\left|\beta_{0}\right|\right) \leq S(1-a \tau)^{s},
$$

and consequently, if

$$
a\left(\frac{1-\tau}{1-a \tau}(1-a \tau)^{-(s-1)} \frac{\left|\alpha_{0}\right|}{1-a}+\sqrt{\frac{\tau(1-\tau)}{1-a \tau}}(1-a \tau)^{-(s-1 / 2)} \frac{\left|\beta_{0}\right|}{\sqrt{1-a}}\right) \leq S .
$$

Since $a, \tau \in[0,1], 1-\tau \leq 1-a \tau$ and $\left|\alpha_{0}\right|+\left|\beta_{0}\right| \leq \sqrt{2}\left|\zeta_{0}\right|$, the last condition will hold if $\sqrt{2}\left|\zeta_{0}\right| \leq S(1-a)^{s}$.

Assuming $L_{0}\left|\zeta_{0}\right| \leq\left(1-\varepsilon_{0}\right)^{s}$ and taking $\rho=h\left(\frac{1+\left(L_{0} \delta\right)^{1 / s}}{1-\left(L_{0} \delta\right)^{1 / s}}\right), h(t)=t+\sqrt{t^{2}-1}$, we have $L_{0}\left|\zeta_{0}\right|=(1-a(\rho))^{s}$; that is, $\psi(\{|z|=1\}) \subset E$. By the maximum principle for subharmonic functions (applied to the domain $\{z \in \mathbb{C}:|z|>1\}$ ), we get

$$
\log V_{E}(\psi(\zeta)) \leq d \log |\zeta|, \quad|\zeta| \geq 1
$$

In particular,

$$
V_{E}\left(x_{0}+\zeta_{0} v\right)=V_{E}(\psi(\rho)) \leq d \log \rho=d \log h\left(\frac{1+\left(L_{0}\left|\zeta_{0}\right|\right)^{1 / s}}{1-\left(L_{0}\left|\zeta_{0}\right|\right)^{1 / s}}\right) .
$$

The inequality $1-\left(L_{0}\left|\zeta_{0}\right|\right)^{1 / s} \geq \varepsilon_{0}$ implies that

$$
h\left(\frac{1+\left(L_{0} \delta\right)^{1 / s}}{1-\left(L_{0} \delta\right)^{1 / s}}\right) \leq h\left(1+\left(2 / \varepsilon_{0}\right)\left(L_{0} \delta\right)^{1 / s}\right) \leq 1+A \delta^{1 /(2 s)},
$$

where

$$
A=\left(2 / \varepsilon_{0}\right)^{1 / 2} L_{0}^{1 /(2 s)}\left(\sqrt{2 / \varepsilon_{0}}+\sqrt{2\left(1-\varepsilon_{0}\right) / \varepsilon_{0}}\right) \leq\left(4 / \varepsilon_{0}\right) L_{0}^{1 /(2 s)}=B .
$$

Since for every $d \geq 1$, the function $\left((1+x)^{d}-1\right) / x$ is increasing for $x>0$, we obtain

$$
\left(1+B\left|\zeta_{0}\right|^{1 /(2 s)}\right)^{d} \leq 1+C_{0}\left|\zeta_{0}\right|^{1 /(2 s)}
$$

where $C_{0}=\max _{r \leq r_{0}}\left(\left(1+B r^{1 /(2 s)}\right)^{d}-1\right) / r^{1 /(2 s)}=\left(\left(1+B r_{0}^{1 /(2 s)}\right)^{d}-1\right) / r_{0}^{1 /(2 s)}$. 
Finally, $V_{E}\left(x_{0}+\zeta_{0} v\right) \leq \log \left(1+C_{0}\left|\zeta_{0}\right|^{1 /(2 s)}\right) \leq C_{0}\left|\zeta_{0}\right|^{1 /(2 s)}$

Applying Corollary 2.10 and Remark 3.1, we get the following result that specifies an earlier result by Pawłucki and Pleśniak (cf. [22, Theorem 2.1]).

Corollary 4.11 If $E \in \operatorname{UPC}_{e_{j}}\left(s_{j}, S_{j}, d_{j}\right), j=1, \ldots, N$, then there exists a constant $B$ such that $E \in \operatorname{HCP}(\gamma, B)$, with $\gamma=1 /\left(2 \min _{j} s_{j}\right)$. In particular, if $E \in$ $\mathrm{UPC}(s, S, d)$, then $E \in \operatorname{HCP}(1 /(2 s), B)$.

\section{Applications of Theorem 2.9 for Disconnected Sets}

The first proposition regards certain onion type sets in the complex plane that are very useful in a problem concerning local and global Markov's properties (see [10]). The Hölder continuity property of these sets does not appear to follow from the main result in [13].

Proposition 5.1 Let $\left(a_{j}\right)_{j}$ be a strictly decreasing sequence of positive numbers such that $a_{1}=1, a_{j} \rightarrow 0$ as $j \rightarrow \infty$, and let $\varphi_{j} \in\left(0, \frac{\pi}{2}\right)$ for $j=1,2, \ldots$ Write

$$
\begin{gathered}
C_{j}:=\left\{a_{j} e^{i t}: t \in\left[\varphi_{j}, 2 \pi\right]\right\} \text { for } j=1,2, \ldots, \\
E:=\{0\} \cup \bigcup_{j=1}^{\infty} C_{j} .
\end{gathered}
$$

If $\left|1-e^{i \varphi_{j}}\right| \leq a_{j+1}$ for $j=1,2, \ldots$, then $E \in \operatorname{HCP}\left(\frac{1}{6}, B\right)$ for some $B>0$.

Proof First, we note that

$$
F:=\left\{e^{i t}: t \in[\pi / 2,2 \pi]\right\}
$$

is a connected compact set, and so $F \in \operatorname{HCP}\left(\frac{1}{2}, B_{F}\right)$, with some constant $B_{F} \geq 1$ (see, e.g., [11, Corollary 2.2]). From implication (8), we see that $F \in \operatorname{VMI}\left(2, M_{F}\right)$, with $M_{F}=\left(e B_{F} / 2\right)^{2}$. We can assume that $M_{F} \geq \max \{2 e, 1 / C(E)\}$.

For any polynomial $P$ of degree at most $n$, for $k \in\{1, \ldots, n\}$ and $z_{0} \in E$, we will prove the inequality

$$
\left|P^{(k)}\left(z_{0}\right)\right| \leq M^{k} \frac{n^{6 k}}{k^{5 k}}\|P\|_{E}, \quad \text { where } M=3 M_{F} \exp \left(3 M_{F}\left(1+e^{3 M_{F}}\right)\right) .
$$

By Theorem 2.9, and since $k^{k} \geq k$ !, condition (14) implies that $E \in \operatorname{HCP}\left(\frac{1}{6}, B\right)$, with $B=6 M^{1 / 6}$. Therefore, the proof is completed by showing (14).

Observe first that for any monic polynomial $P$ of degree $n$ and for $k=n$, we have

$$
\left\|P^{(k)}\right\|_{E}=\left(\frac{1}{C(E)}\right)^{k} n !(C(E))^{n} \leq\left(\frac{1}{C(E)}\right)^{k} n !\|P\|_{E},
$$


because in $\mathbb{C}, C(E)$ is equal to the Chebyshev constant of $E$. Consequently,

$$
\left\|P^{(k)}\right\|_{E} \leq\left(M_{F}\right)^{k} \frac{n^{6 k}}{k^{5 k}}\|P\|_{E}
$$

for all polynomials of degree at most $n$ not necessarily monic. Thus condition (14) is fulfilled for $k=n$.

Now consider $k<n$. We first examine $z_{0}=0$, and we will show that

$$
\left|P^{(k)}(0)\right| \leq\left(\frac{3 M_{F} e^{3 M_{F}} n^{4}}{k^{3}}\right)^{k}\|P\|_{E} .
$$

For this purpose, find $j \in \mathbb{N}$ such that $\frac{1}{a_{j}} \leq \frac{n^{2}}{k^{2}}<\frac{1}{a_{j+1}}$. By Cauchy's integral formula,

$$
\left|P^{(k)}(0)\right| \leq \frac{1}{a_{j}}\left\|P^{(k-1)}\right\|_{C\left(0, a_{j}\right)},
$$

where $C\left(0, a_{j}\right)$ is the circle with the radius $a_{j}$ about the origin. The norm of $P^{(k-1)}$ on $C\left(0, a_{j}\right)$ is attained at some point $w_{0} \in C\left(0, a_{j}\right)$. Define $F_{j}:=a_{j} F$. Obviously, $F_{j} \in \operatorname{VMI}\left(2, M_{F} / a_{j}\right)$. If $w_{0} \in F_{j}$, we have

$$
\begin{aligned}
\left|P^{(k)}(0)\right| & \leq \frac{1}{a_{j}}\left\|P^{(k-1)}\right\|_{F_{j}} \leq\left(\frac{1}{a_{j}}\right)^{k} \frac{\left(M_{F} n^{2}\right)^{k-1}}{(k-1) !}\|P\|_{F_{j}} \\
& \leq\left(\frac{1}{a_{j}}\right)^{k}\left(\frac{3 M_{F} n^{2}}{k-1}\right)^{k-1}\|P\|_{F_{j}} \\
& \leq\left(\frac{n^{2}}{k^{2}}\right)^{k}\left(\frac{3 M_{F} n^{2}}{k}\right)^{k}\|P\|_{F_{j}} \leq\left(\frac{3 M_{F} n^{4}}{k^{3}}\right)^{k}\|P\|_{E} \quad \text { for } k \geq 2,
\end{aligned}
$$

because $t \mapsto\left(\frac{M_{F} n^{2}}{t}\right)^{t}$ is an increasing function whenever $t \in(0, n+1] \subset\left(0, \frac{M_{F} n^{2}}{e}\right]$ and $F_{j} \subset C_{j} \subset E$. The case of $k=1$ is easy to verify, and thus inequality (15) is fulfilled for all $k \in\{1, \ldots, n\}, w_{0} \in F_{j}$.

If $w_{0} \in C\left(0, a_{j}\right) \backslash F_{j}$, by Taylor's formula and VMI for $F$, we get

$$
\begin{aligned}
\left|P^{(k)}(0)\right| & \leq \frac{1}{a_{j}}\left|P^{(k-1)}\left(w_{0}\right)\right| \leq \frac{1}{a_{j}} \sum_{l=0}^{n-k+1} \frac{1}{l !}\left|P^{(k-1+l)}\left(a_{j}\right)\right|\left|a_{j}-w_{0}\right|^{l} \\
& \leq \frac{1}{a_{j}} \sum_{l=0}^{n-k+1} \frac{1}{l !}\left(\frac{1}{a_{j}}\right)^{k-1+l}\left(\frac{3 M_{F} n^{2}}{k-1+l}\right)^{k-1+l}\|P\|_{F_{j}} a_{j}^{l}\left|1-e^{i \varphi_{j}}\right|^{l} \\
& \leq\left(\frac{1}{a_{j}}\right)^{k} \sum_{l=0}^{n-k+1} \frac{1}{l !}\left(\frac{3 M_{F} n^{2}}{k+l}\right)^{k+l} a_{j+1}^{l}\|P\|_{F_{j}},
\end{aligned}
$$


the last inequality being a consequence of the assumption of Proposition 5.1. Since $F_{j} \subset E$ and $\frac{1}{a_{j}} \leq \frac{n^{2}}{k^{2}}<\frac{1}{a_{j+1}}$, we can write

$$
\begin{aligned}
\left|P^{(k)}(0)\right| & \leq\left(\frac{n^{2}}{k^{2}}\right)^{k}\left(\frac{3 M_{F} n^{2}}{k}\right)^{k n-k+1} \sum_{l=0}^{n} \frac{1}{l !}\left(\frac{3 M_{F} n^{2}}{k}\right)^{l}\left(\frac{k^{2}}{n^{2}}\right)^{l}\|P\|_{E} \\
& \leq\left(\frac{3 M_{F} e^{3 M_{F}} n^{4}}{k^{3}}\right)^{k}\|P\|_{E},
\end{aligned}
$$

and this yields inequality (15).

We now turn to the case $z_{0} \neq 0$. Clearly, $z_{0} \in C_{j}$ for some $j \in\{1,2, \ldots$,$\} . If$ $a_{j} \leq \frac{k^{4}}{n^{4}}$, then by (15), we have

$$
\begin{aligned}
\left|P^{(k)}\left(z_{0}\right)\right| & \leq \sum_{l=0}^{n-k} \frac{1}{l !}\left|P^{(k+l)}(0)\right|\left|z_{0}\right|^{l} \leq \sum_{l=1}^{n} \frac{1}{l !}\left(\frac{3 M_{F} e^{3 M_{F}} n^{4}}{(k+l)^{3}}\right)^{k+l}\|P\|_{E} a_{j}^{l} \\
& \leq\left(\frac{3 M_{F} e^{3 M_{F}} n^{4}}{k^{3}}\right)^{k} \sum_{l=1}^{n} \frac{1}{l !}\left(\frac{3 M_{F} e^{3 M_{F}} n^{4}}{k^{3}}\right)^{l}\left(\frac{k^{4}}{n^{4}}\right)^{l}\|P\|_{E} \\
& \leq\left(\frac{3 M_{F} e^{3 M_{F}} \exp \left(3 M_{F} e^{3 M_{F}}\right) n^{4}}{k^{3}}\right)^{k}\|P\|_{E},
\end{aligned}
$$

and (14) is proved in this case.

It remains to show estimate (14) if $a_{j}>\frac{k^{4}}{n^{4}}$. Let $F_{j}^{\prime}$ be a set obtained by a rotation of $F_{j}$ about the origin such that $z_{0} \in F_{j}^{\prime} \subset C_{j}$. Since $F_{j}^{\prime} \in \operatorname{VMI}\left(2, M_{F} / a_{j}\right)$, we have

$$
\left|P^{(k)}\left(z_{0}\right)\right| \leq\left\|P^{(k)}\right\|_{F_{j}^{\prime}} \leq \frac{1}{a_{j}^{k}}\left(\frac{3 M_{F} n^{2}}{k}\right)^{k}\|P\|_{F_{j}^{\prime}} \leq\left(\frac{n^{4}}{k^{4}}\right)^{k}\left(\frac{3 M_{F} n^{2}}{k}\right)^{k}\|P\|_{E},
$$

and (14) is proved at every point $z_{0} \in E$.

The second example presents the application of Theorem 2.9 for certain compact sets consisting of infinitely many pairwise disjoint subsets of $\mathbb{C}^{N}$.

Proposition 5.2 Let $\mu \geq 2, b \in(0, \sqrt{2}-1)$, and let $\left(a_{j}\right)_{j},\left(r_{j}\right)_{j}$ be sequences of positive numbers such that

$$
a_{1}=2, \quad r_{1}=1, \quad a_{j}=r_{j}+r_{j}^{2}, \quad r_{j}=b r_{j-1}^{\mu} \quad \text { for } j \geq 2 .
$$

Then the set $E$ defined by

$$
\begin{aligned}
& E:=\{0\} \cup \bigcup_{j=1}^{\infty} E_{j}, \\
& E_{j}:=\left\{z=\left(z_{1}, \ldots, z_{N}\right) \in \mathbb{C}^{N}:\left|z_{1}-a_{j}\right| \leq r_{j},\left|z_{2}\right| \leq r_{j}, \ldots,\left|z_{N}\right| \leq r_{j}\right\}
\end{aligned}
$$

satisfies $E \in \operatorname{HCP}\left(\frac{1}{2+\mu}, B\right)$, with some $B>0$. 
Proof Fix $n \in\{1,2, \ldots\}$ and a polynomial $P$ of degree at most $n$. As a first step, we shall show that for each $\alpha \in \mathbb{N}_{0}^{N},|\alpha| \leq n$,

$$
\left|D^{(\alpha)} P(0)\right| \leq\left(\frac{e^{N}}{b}\right)^{|\alpha|}\left(\frac{n^{1+\mu}}{|\alpha|^{\mu}}\right)^{|\alpha|}\|P\|_{E} .
$$

For this purpose, find $j \geq 2$ such that $r_{j}<\frac{|\alpha|}{n} \leq r_{j-1}$, where $|\alpha| \leq n$ is fixed. From (7), we have

$$
\left\|D^{\alpha} P\right\|_{E_{j}} \leq \frac{n^{|\alpha|}}{r_{j}^{|\alpha|}}\|P\|_{E_{j}}
$$

and thus, by Theorem 2.9 and Example 2.6, $E_{j} \in \operatorname{HCP}\left(1, \frac{N}{r_{j}}\right)$. In particular, we get

$$
V_{E_{j}}(0) \leq \frac{N}{r_{j}} \operatorname{dist}\left(0, E_{j}\right)=\frac{N}{r_{j}}\left(a_{j}-r_{j}\right)=N r_{j}<\frac{|\alpha| N}{n} .
$$

Formula (3) leads us to

$$
\left|D^{(\alpha)} P(0)\right| \leq\left(e^{V_{E_{j}}(0)}\right)^{n-|\alpha|}\left\|D^{\alpha} P\right\|_{E_{j}} .
$$

By the above, it follows that

$$
\left|D^{(\alpha)} P(0)\right| \leq e^{|\alpha| N} \frac{n^{|\alpha|}}{r_{j}^{|\alpha|}}\|P\|_{E_{j}} \leq e^{|\alpha| N} \frac{n^{|\alpha|+\mu|\alpha|}}{\left(b|\alpha|^{\mu}\right)^{|\alpha|}}\|P\|_{E},
$$

and inequality (16) is proved.

Now consider $z_{0} \in E \backslash\{0\}$ and $|\alpha| \leq n$. If $z_{0} \in E_{j}$ and $r_{j} \geq\left(\frac{|\alpha|}{n}\right)^{1+\mu}$, then

$$
\left|D^{(\alpha)} P\left(z_{0}\right)\right| \leq \frac{n^{|\alpha|}}{r_{j}^{|\alpha|}}\|P\|_{E_{j}} \leq \frac{n^{2|\alpha|+\mu|\alpha|}}{|\alpha|^{(1+\mu)|\alpha|}}\|P\|_{E} .
$$

In the case of $r_{j}<\left(\frac{|\alpha|}{n}\right)^{1+\mu}$, Taylor's formula and inequality (16) yield

$$
\begin{aligned}
\left|D^{(\alpha)} P\left(z_{0}\right)\right| \leq & \sum_{|\beta| \leq n-|\alpha|} \frac{1}{\beta !}\left|D^{\alpha+\beta} P(0)\right|\left\|z_{0}\right\|_{2}^{|\beta|} \\
\leq & \sum_{|\beta| \leq n-|\alpha|} \frac{1}{\beta !}\left(\frac{e^{N}}{b}\right)^{|\alpha|+|\beta|}\left(\frac{n^{1+\mu}}{(|\alpha|+|\beta|)^{\mu}}\right)^{|\alpha|+|\beta|}\|P\|_{E}\left(r_{j} \sqrt{N+8}\right)^{|\beta|} \\
\leq & \left(\frac{e^{N}}{b}\right)^{|\alpha|}\left(\frac{n^{1+\mu}}{|\alpha|^{\mu}}\right)^{|\alpha|}\|P\|_{E} \\
& \times \sum_{l=0}^{n-|\alpha|} \frac{N^{l}}{l !}\left(\frac{e^{N} \sqrt{N+8}}{b}\right)^{l}\left(\frac{n^{1+\mu}}{|\alpha|^{\mu}}\right)^{l}\left(\frac{|\alpha|}{n}\right)^{(1+\mu) l}
\end{aligned}
$$




$$
\begin{aligned}
& \leq\left(\frac{1}{b} e^{N+N \sqrt{N+8} e^{N} / b}\right)^{|\alpha|}\left(\frac{n^{1+\mu}}{|\alpha|^{\mu}}\right)^{|\alpha|}\|P\|_{E} \\
& \leq\left(\frac{1}{b} e^{N+N \sqrt{N+8} e^{N} / b}\right)^{|\alpha|} \frac{\left(n^{1+\mu}\right)^{|\alpha|}}{(|\alpha| !)^{\mu}}\|P\|_{E} .
\end{aligned}
$$

Hence, and from inequalities $(16,17)$, we conclude that $E \in \operatorname{VMI}(2+\mu$, $\left.\frac{1}{b} e^{N+N \sqrt{N+8} e^{N} / b}\right)$, and Theorem 2.9 leads to $E \in \operatorname{HCP}\left(\frac{1}{2+\mu}, B\right)$, with $B=$ $\left(\frac{N}{b} e^{N+N \sqrt{N+8} e^{N} / b}\right)^{\frac{1}{2+\mu}}(2+\mu)$, which proves the assertion.

Remark 5.3 We close this paper by offering two questions for further research:

1. Does the continuity of the pluricomplex Green's function $V_{E}$ with respect to each variable separately imply the L-regularity of $E$ ?

2. Consider a Markov set $E \subset \mathbb{C}^{N}$ and its pluricomplex Green function $V_{E}$. Is the function $V_{E}$ continuous with respect to each variable separately?

For the real univariate case, the answer to the second question is positive, because if $E \subset \mathbb{R}$ is a Markov set, then it is L-regular (see [9]).

Open Access This article is distributed under the terms of the Creative Commons Attribution License which permits any use, distribution, and reproduction in any medium, provided the original author(s) and the source are credited.

\section{References}

1. Andrievski, V.V.: The highest smoothness of the Green function implies the highest density of a set. Ark. Mat. 42, 217-238 (2004)

2. Baran, M.: Plurisubharmonic extremal function and complex foliation for a complement of a convex subset of $\mathbb{R}^{n}$. Mich. Math. J. 39, 395-404 (1992)

3. Baran, M.: Markov inequality on sets with polynomial parametrization. Ann. Pol. Math. 60, 69-79 (1994)

4. Baran, M.: Cauchy-Poisson transform and polynomial inequalities. Ann. Pol. Math. 95, 199-206 (2009)

5. Baran, M., Bialas-Ciez, L.: Product property for capacities in $\mathbb{C}^{N}$. Ann. Pol. Math. 106, 19-29 (2012)

6. Baran, M., Bialas-Ciez, L., Milówka, B.: On the best exponent in Markov inequality. Potential Anal. 38, 635-651 (2013)

7. Bialas-Ciez, L.: Markov sets in $\mathbb{C}$ are not polar. Bull. Pol. Acad. Sci., Math. 46, 83-89 (1998)

8. Bialas-Ciez, L.: Siciak's extremal function via Bernstein and Markov constants for compact sets in $\mathbb{C}^{N}$. Ann. Pol. Math. 106, 41-51 (2012)

9. Bialas-Ciez, L., Eggink, R.: L-regularity of Markov sets and of m-perfect sets in the complex plane. Constr. Approx. 27, 237-252 (2008)

10. Bialas-Ciez, L., Eggink, R.: Equivalence of the global and local Markov inequalities in the complex plane, submitted

11. Bialas-Ciez, L., Kosek, M.: How to construct totally disconnected Markov sets? Ann. Mat. Pura Appl. (4) 190, 209-224 (2011)

12. Bos, L., Calvi, J.-P., Levenberg, N.: On the Siciak extremal function for real compact convex sets. Ark. Mat. 39, 245-262 (2001)

13. Carleson, L., Totik, V.: Hölder continuity of Green's functions. Acta Sci. Math. 70, 557-608 (2004)

14. Goetgheluck, P.: Markov's inequality on locally Lipschitzian compact subsets of $R^{N}$ in $L^{p}$-spaces. J. Approx. Theory 49, 303-310 (1987)

15. Klimek, M.: Pluripotential Theory. London Mathematical Society Monographs New Series, vol. 6. Clarendon Press, Oxford (1991) 
16. Kosek, M.: Hölder continuity property of filled-in Julia sets in $\mathbb{C}^{n}$. Proc. Am. Math. Soc. 125, 20292032 (1997)

17. Kosek, M.: Hölder continuity property of composite Julia sets. Bull. Pol. Acad. Sci., Math. 46, 391399 (1998)

18. Kosek, M.: Hölder exponents for the pluricomplex Green function of Julia type sets. Indian J. Pure Appl. Math. 42, 475-492 (2011)

19. Kosek, M.: Hölder exponents of the Green functions of planar polynomial Julia sets. Ann. Mat. Pura Appl. (2012). doi:10.1007/s10231-012-0278-6

20. Lelong, P., Gruman, L.: Entire Functions of Several Complex Variables. Springer, Berlin (1986)

21. Milówka, B.: Markov's inequality and a generalized Pleśniak condition. East J. Approx. 11, 291-300 (2005)

22. Pawłucki, W., Pleśniak, W.: Markov's inequality and $\mathcal{C}^{\infty}$ functions on sets with polynomial cusps. Math. Ann. 275, 467-480 (1986)

23. Pierzchała, R.: UPC condition in polynomially bounded o-minimal structures. J. Approx. Theory 132, 25-33 (2005)

24. Pierzchała, R.: Siciak's extremal function of non-UPC cusps. I. J. Math. Pures Appl. 94, 451-469 (2010)

25. Pleśniak, W.: Markov's inequality and the existence of an extension operator for $C^{\infty}$ functions. J. Approx. Theory 61(1), 106-117 (1990)

26. Ransford, T., Rostand, J.: Hölder exponents of Green's functions of Cantor sets. Comput. Methods Funct. Theory 8, 151-158 (2008)

27. Shadrin, A.: Twelve proofs of the Markov inequality. In: Approx. Theory: A Volume Dedicated to Borislav Bojanov, pp. 233-298. Prof. Drinov Acad. publ. House, Sofia (2004)

28. Siciak, J.: Extremal plurisubharmonic functions in $\mathbb{C}^{N}$. Ann. Pol. Math. 39, 175-211 (1981)

29. Siciak, J.: Wiener's type sufficient conditions in $C^{n}$. Univ. Iagel. Acta Math. 35, 151-161 (1997) 